\title{
Signal Resampling Technique Combining Level Crossing and Auditory Features
}

\author{
Nagesha* and G Hemantha Kumar \\ Dept of Studies in Computer Science, University of Mysore, \\ Mysore - 570 006, India \\ shan_bk@yahoo.com
}

\begin{abstract}
Level crossing based sampling might be used as an alternative to Nyquist theory based sampling of a signal. Level crossing based approach take advantage of statistical properties of the signal, providing cues to efficient nonuniform sampling. This paper presents new threshold level allocation schemes for level crossing based nonuniform sampling. Intuitively, it is more reasonable if the information rich regions of the signal are sampled finer and those with sparse information are sampled coarser. To achieve this objective, we proposed non-linear quantization functions which dynamically assign the number of quantization levels depending on the importance of the given amplitude range. Various aspects of proposed techniques are discussed and experimentally validated. Its efficacy is investigated by comparison with Nyquist based sampling.
\end{abstract}

\section{Introduction}

The concept of level crossing sampling scheme has been proposed for analog to digital conversion [3. Several case studies in analog to digital converters shows that level crossing based sampling technique can be more effective than existing synchronous Nyquist analog to digital converters (ADC). The level crossing sampling scheme has also demonstrated for speech applications using CMOS technology and a voltage mode approach for the analog parts of the converter 4. Electrical simulations proved that the Figure of Merit of asynchronous level crossing converters increased compared to synchronous Nyquist ADCs. Level crossing sampling scheme has been suggested in literature for bursty signals, non-bandlimited signal [5] and band limited Gaussian random processes [6].

Conventional Nyquist based signal sampling is with uniform time-step variable amplitude. The new class of nonuniform sampling approach has been used to improve the performance of Nyquist ADCs exploiting the inner statistical properties of the signal. Several signals has interesting statistical properties, but Nyquist based sampling do not take the advantage of them. Signals such as electro cardiograms, speech signals, temperature sensors, pressure sensors are almost always constant and may vary significantly during brief moments. Due to Nyquist theory, which is to ensure the sampling to be at least twice that of the

^ Corresponding author: 0821-2510789.

A. Ghosh, R.K. De, and S.K. Pal (Eds.): PReMI 2007, LNCS 4815, pp. 447454, 2007.

(C) Springer-Verlag Berlin Heidelberg 2007 
input signal frequency bandwidth, it is obvious that, in the time domain, this condition may result in a large number of samples with redundant information. It has been proved in 14 that level crossing sampling approach can lead to reduction in number of samples.

Level crossing scheme sends a pulse whenever the source signal crosses a threshold level. The threshold levels are uniformly distributed in the amplitude range [1/3|4 5]. The uniform threshold step size used in the level crossing regardless of the signal amplitude characteristics. The linear threshold allocation will result in a higher SNR at the region of higher amplitude than the region of lower amplitude. Hence, increased SNR at the higher signal amplitude does not increase the perceived audio quality because humans are most sensitive to lower amplitude components. To exploit this factor, a nonuniform threshold level allocation schemes based on Incomplete Beta Function (IBF), logarithmic and linear function are proposed in this paper. The parameters of the IBF are chosen in such a way that it focuses on sensitive lower amplitude regions.

The paper is organized as follows. Section 2 describes the level crossing based sampling approach with the proposed nonuniform threshold allocation scheme. Section 3 discusses the incorporation of IBF, logarithmic and linear functions to formulate a rule for allocation of nonuniform threshold levels in multilevel crossing. Section 4 explains the experimentation setup for testing the proposed approach and gives the results and analysis. Section 5 is devoted to major conclusions as well as gives directions for future lines of work.

\section{Irregular Sampling Model}

Level Crossing Analysis represents an approach to interpretation and characterization of time signals by relating frequency and amplitude information. Measurement of level crossing of a signal is defined as the crossings of a threshold level $l$ by consecutive samples.

The level sampling with a level allocation function $f(s)$ is the mapping $L_{f(s)}$ : $R \rightarrow f(s) Z: L_{f(s)}=f(s)\lfloor s / f(f(s))\rfloor$ where $L=\left(l_{1}, l_{2}, \cdots, l_{N}\right)$ set of nonuniform spaced levels.

Since the quantization levels are irregularly spaced across the amplitude range of the signal, it increases the efficiency of bit usage. The spacing of the levels is decided by the importance of the amplitude segments which is discussed in section 3. A sample is recorded when the input signal crosses one of the nonuniformly spaced levels. The precession of time of the recorded sample is decided by the local timer $T$.

Let $L=\left(l_{1}, l_{2}, \ldots, l_{N}\right)$ be the set of nonuniform spaced levels and $2^{b}-1=N$ quantization levels with $b$ bit resolution.

Definition 2.1: The level crossing of the threshold level $l \in L$ by signal $s(t)$ with period $T$ is given by

$$
L_{f(s)}\left(s, I_{n i}\right)=l \quad \text { iff } s\left[\frac{i-1}{n} T\right]\left[s\left[\frac{i-1}{n} T\right]-l\right]<0
$$


where $n$ subintervals are defined by $I_{n i}=\left[\frac{i-1}{n} T, \frac{i}{n} T\right], i=1,2, \cdots, n$. If a sample is recorded and transmitted every time a level crossing occurs, the encoding procedure is called asynchronous delta modulation [2].

\section{Rules for Irregular Sampling}

Determining the threshold levels is very important as it has a huge impact on the performance of coding. Unfortunately there is no theory available to determine those values. The uniform threshold levels are not the efficient coding of the levels because they do not take advantage of the statistical properties of the signal. The present study considers nonuniform threshold level allocation scheme depending on the signal amplitude characteristics and rules which controls the importance of amplitude regions. As a result, signals with lesser activity in higher amplitude regions compared to the lower amplitude regions, will have less number of levels at higher amplitude region. In this section, IBF, logarithmic and linear function rules are analyzed.

\subsection{Incomplete Beta Function}

The lack of data to decide the exact number of levels for a given amplitude range, creates problems concerning the selection of number of levels. In such cases, an expert will have to assume the levels. For this reason, the flexible incomplete beta distribution, capable of attaining a variety of shapes could be used in Level Crossing applications. Because of its extreme flexibility, the distribution appears ideally suited for the computation of number of levels for a specific amplitude region of a signal. The Incomplete beta function $I(z, \alpha, \beta)$ is defined by [7]:

$$
I(z, \alpha, \beta) \equiv \frac{1}{B(\alpha, \beta)} \int_{0}^{z} u^{\alpha-1}(1-u)^{\beta-1} d u \quad \alpha>0, \beta>0,0 \leq z \leq 1
$$

Eq, 2 has the limiting values $I_{0}(\alpha, \beta)=0, I_{1}(\alpha, \beta)=1$. The shape of the incomplete beta function obtained from Eq2 depends on the choice of its two parameters $\alpha$ and $\beta$. Estimating these parameters is a challenge since these parameters control the number of levels for a given amplitude range of a speech signal along with the signal probability density function (PDF). We have empirically chosen the values of $\alpha$ and $\beta$ such that IBF gives more weight to amplitude regions of importance.

\subsection{Linear Function}

Although human auditory perception is certainly does not use a linear function, this group of mapping methods renders acceptable results for a wide range of applications. Its strength is its simplicity and speed. The linear function is defined by

$$
\operatorname{linear}(n)=n
$$


However, computing the importance of amplitude regions in linear scale is not merely a mater of mathematical convenience. There is more compelling, physical consideration to be taken into account, related to the importance of amplitude regions. Natural worst case representation for characterization of physical systems is linear. This means that all amplitude regions become equally important.

\subsection{Logarithmic Function}

A logarithmic of a number $x$ in base $b$ is a number $n$ such that $x=b^{n}$, where the value $b$ must be neither 0 nor a root of 1 . It is usually written as

$$
\log _{b}(x)=n
$$

When $x$ and $b$ are further restricted to positive real numbers, the logarithm is a unique real number.

Representation of importance of amplitude on a logarithmic scale can be helpful when the importance of regions varies slowly. Logarithmic rule assigns less number of levels to the corner amplitude regions and more levels are assigned logarithmically in important amplitude regions. The center amplitude regions (near zero amplitude regions) are considered to be important amplitude regions. This issue, however is not whether to accept or reject logarithmic rule but to appreciate where it fits in, and where it does not.

\subsection{Level Estimation}

In a deterministic environment, the accuracy of the signal reconstruction depends on several parameters such as positioning of the levels, total number of levels, statistical properties of the signal etc. Specifically, for a given signal we analyze the structural behavior by estimating its PDF. The signal histogram is approximated to obtain the signal PDF $p(x)$.

Now consider a signal with PDF $p(x)$ and level allocation rule $R(x)$. Let $N$ be the total number of levels. The locations of $N$ levels are estimated by

$$
L(x)=p(x) \otimes R(x)
$$

The $\otimes$ symbol represents linear filtering. $L(x)$ gives the probability of distribution of levels and it guides the distribution of $N$ levels over the amplitude range. As expected, the spacing of $N$ levels are not uniform they are nonuniformly spaced over the amplitude range. Each level can be represented with $\log _{2}(N)$ bits. Hence only amplitude regions with high activity will be allocated more number of levels using the rule $R(x)$ and signal amplitude PDF.

\section{Experimental Evaluation}

In this section, the performance of the proposed approach is evaluated for speech signals. We have run simulations for the level crossing based sampling of speech 

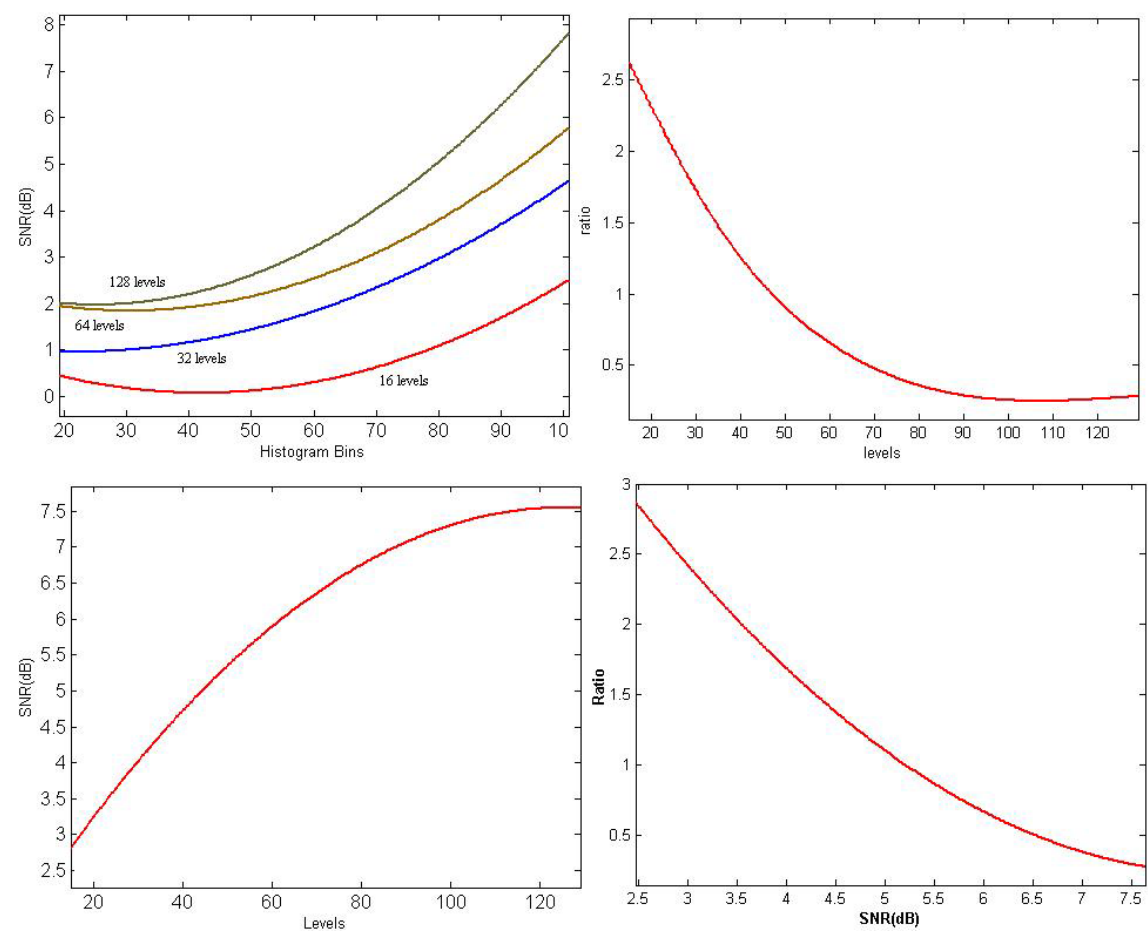

Fig. 1. Experimental results for IBF (a) Histogram bin versus SNR. (b) Quantization level versus ratio (c) Quantization level versus SNR.(d) SNR versus Compression ratio.

signals from TIMIT database. The TIMIT speech signals are sampled by $16 \mathrm{KHz}$ sampling rate and each sample size is 16 bit. The PDF of the speech signal is estimated by computing the amplitude histogram of the signal with 100 bins. The total numbers of quantization levels needed to sample the given signal are set to 16, 32, 64 and 128. The accuracy of distribution of the levels computed from Eq.5, also depends on the number of bins used to compute convoluted PDF of the signal. The levels are estimated for 20, 40, 60, 80, 100 bins for comparison and analysis. We evaluated the system with proposed rules IBF, logarithmic and linear functions.

By comparing IBF, logarithmic and linear rule results, we analyze the performances. SNR of the resampled signal generally improves as the bins increase for all the levels. The IBF rule gives high SNR consistently compared to the logarithmic and linear rule at all bins. The performance of logarithmic rule is slightly less than that of IBF rule for all the levels (Fig.1(a) and Fig.3(a)). The best performance is observed for IBF rule with 128 levels. In case of linear rule, $1 \mathrm{~dB}$ drop in SNR is observed compared to IBF for 128 levels. Similarly, higher SNR is achieved for IBF and logarithmic rule compared to linear rule at all levels (Fig.1(a), Fig.2(a) and Fig.3(a)). 

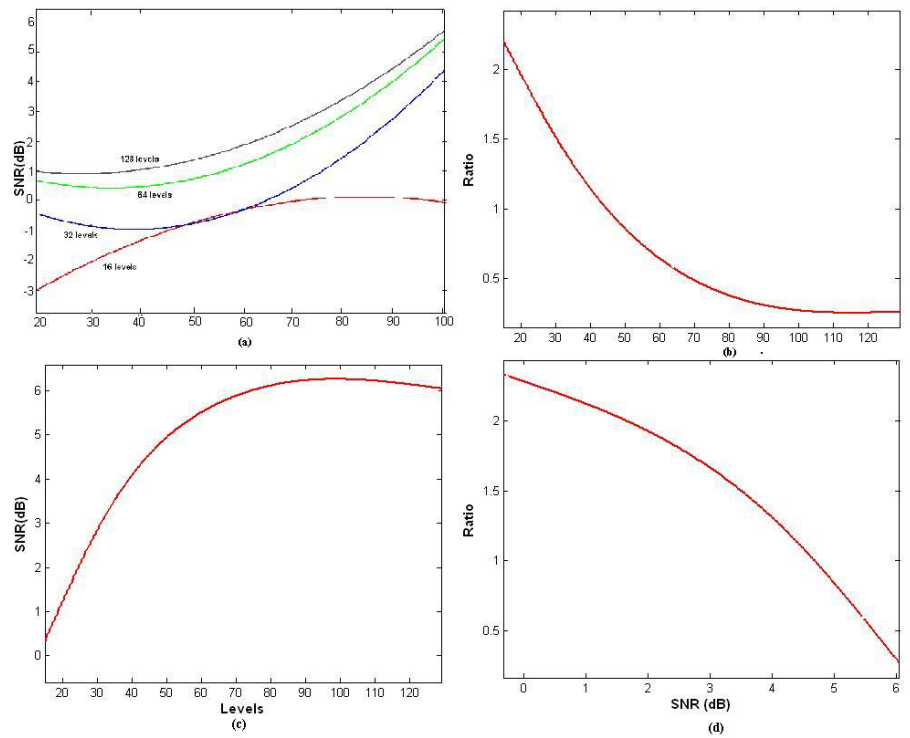

Fig. 2. Experimental results for linear function (a) Histogram bin versus SNR. (b) Quantization level versus ratio (c) Quantization level versus SNR. (d) SNR versus Compression ratio.

The comparison of compression ratio at various levels for the three rules is shown in Fig.1(b), Fig.2(b) and Fig.3(b). We observe that logarithmic rule slightly outperforms IBF rule. The logarithmic rule gives higher SNR for lesser levels and the ratio decreases as the levels are increased. Linear rule results in low compression ratio for all levels. For higher levels all the rules give similar results. The results of SNR versus levels (Fig.1(c), Fig.2(c) and Fig.3(c)) show that IBF and logarithmic rule performance is superior to linear rule at all levels. It is observed that IBF and logarithmic rules produce almost similar performance results. Minimum SNR for 16 levels is near $3 \mathrm{~dB}$ in IBF and logarithmic rule whereas minimum SNR in linear rule for 16 levels is 0.4 dB. Fig.1(d), Fig.2(d) and Fig.3(d) shows the plot of SNR versus compression ratio. The characteristic curve appears to be concave for IBF rule, linear for logarithmic rule and convex for linear rule. Performance of IBF and logarithmic rules are considerably better than linear rule, with higher SNR for higher compression ratio, which is nonetheless better performance. Comparison of IBF, logarithmic and linear rule shows that, the IBF rule and logarithmic outperforms linear rule. Also, performances of IBF slightly outperforms logarithmic rule in compression ratio and SNR.

The behavioral patterns of IBF, logarithmic and linear rule appear to be similar except in SNR versus ratio analysis. IBF rule is based on the auditory properties of the humans. IBF rule distributes more levels in the critical amplitude regions. Similar to IBF, logarithmic rule also considers that near zero amplitude regions are important than the corner amplitude regions. The priority 

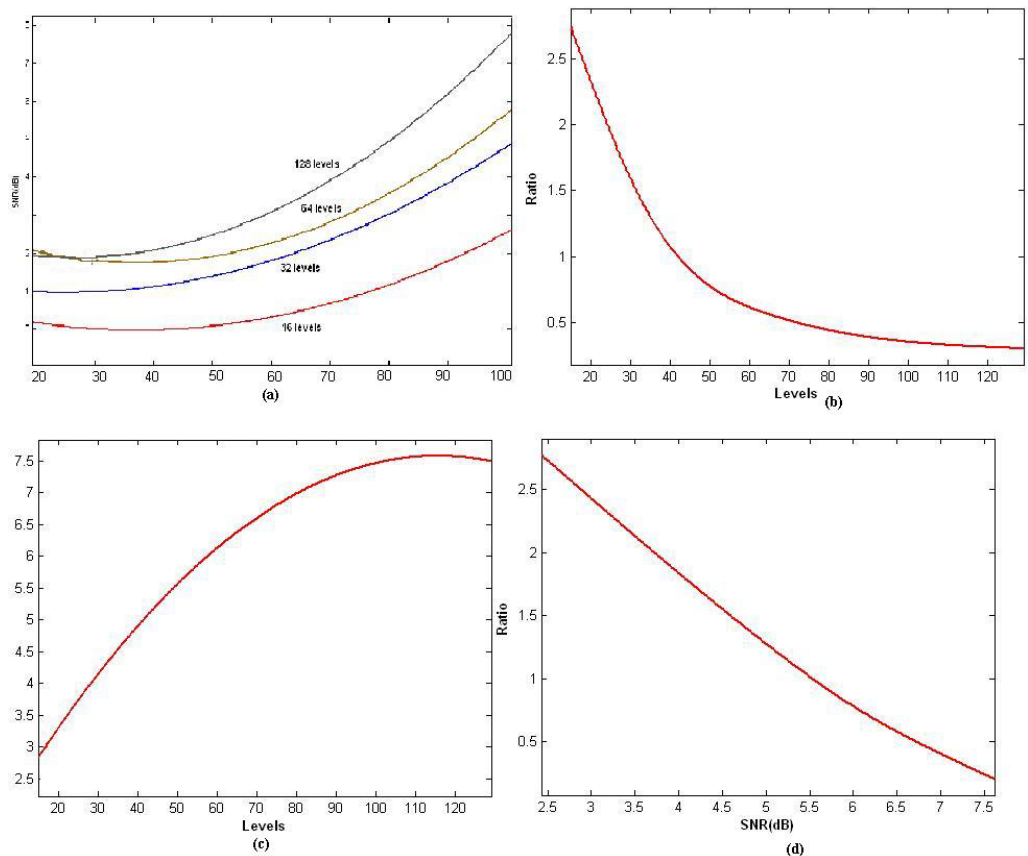

Fig. 3. Experimental results for logarithmic function (a) Histogram bin versus SNR. (b) Quantization level versus ratio (c) Quantization level versus SNR. (d) SNR versus Compression ratio.

of the amplitude regions varies logarithmically from corner amplitude regions to near zero value amplitude regions. Linear rule considers that each amplitude region is equally important. Hence, the SNR of the resampled signal remains consistently superior to linear rule. Lack of levels at critical amplitude regions of the signal decreases the SNR of the resampled signal. The performance of the proposed approaches is fairly consistent with that of Sayiner [3]. This experimental analysis illustrates that signal with special statistical behavior such as speech, medical signals are unsuitable for uniform sampling. These types of signals can be more efficiently sampled using a level crossing scheme.

\section{Conclusion}

In summary, this paper presents new threshold level allocation schemes for level crossing based nonuniform sampling which dynamically assigns the number of quantization levels depending on the importance of the given amplitude range of the input signal. Proposed methods take the advantage of statistical properties of the signal and allocate the nonuniformly spaced quantization levels across the amplitude range. The proposed level allocation scheme for nonuniform sampling based on level crossing may motivate directed attempts to augment traditional 
methods that will improve their ability. Overall, these results motivate continued work on level crossing based nonuniform sampling for improving sampling performance and analysis the signals as a whole.

\section{Acknowledgement}

The authors profoundly thank Mr.Noushath and Mr.Aradhya, Research Scholars, University of Mysore, for helping them to typeset the manuscript in $\mathrm{IT}_{\mathrm{E} X}$.

\section{References}

1. Mitchell, R.J., Gonzalez, R.C.: Multilevel crossing rates for automated signal classification. In: Proc. of ICASSP 1978, vol. 3, pp. 218-222 (April 1978)

2. Inose, H., Aoki, T., Wantanable, K.: Asynchronous delta modulation systems, Electron. Commun., Japan, pp. 34-42 (March 1966)

3. Sayiner, N., Sorensen, H.V., Viswanathan, T.R.: A level crossing sampling scheme for A/D conversion. IEEE Transactions on Circuits and Systems II 43, 335-339 (1996)

4. Allier, E., Sicard, G., Fesquet, L., Renaudin, M.: Asynchronous level crossing analog to digital converters. Measurement Journal 37, 296-309 (2005)

5. Guan, K., Singer, A.C.: A Level Crossing Sampling Scheme for Non-Bandlimited Signals. In: Proc. of ICASSP 2006, vol. 3, pp. III-381-383 (May 2006)

6. Miskowicz, M.: Efficiency of Level-Crossing Sampling for Bandlimited Gaussian Random Processes. In: Proc. of IEEE International Workshop on Factory Communication Systems-2006, pp. 137-142 (June 2006)

7. Press, W.H., Teukolsky, S.A., William, T.V., Brian, P.F.: Numerical Recipes in $\mathrm{C}++$, 2nd edn. Cambridge university press, Cambridge (2002) 